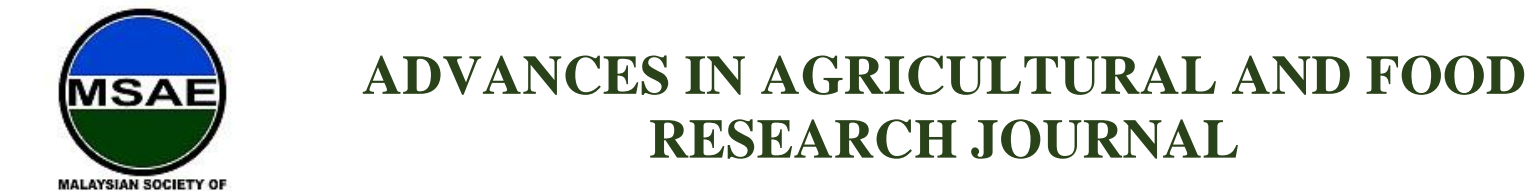

Original Research Article

\title{
Evaluation of Total Phenolic Content and Antioxidant Activities from Different Extraction Techniques of Helianthus tuberosus
}

\begin{abstract}
Nur Diyana Alyas ${ }^{1 *}$, Fadzilah Puteh ${ }^{1}$, Nurin Izzati Mohd Zulkifli², Noor Zafira Noor Hasnan ${ }^{3}$
${ }^{1}$ Enzyme \& Fermentation Technology Programme, Food Science \& Technology Research Centre, MARDI, Persiaran MARDI-UPM, 43400 Serdang, Selangor, Malaysia

${ }^{2}$ Biological Control Programme, Agrobiodiversity \& Environment Research Centre, MARDI, Persiaran MARDI-UPM, 43400 Serdang, Selangor, Malaysia

${ }^{3}$ Department of Process and Food Engineering, Faculty of Engineering, Universiti Putra Malaysia, 43400 Serdang, Selangor, Malaysia

*Corresponding author: Noor Zafira Noor Hasnan, Department of Process and Food Engineering, Faculty of Engineering, Universiti Putra Malaysia, 43400 Serdang, Selangor, Malaysia; noorzafira@upm.edu.my
\end{abstract}

\begin{abstract}
This study investigated the difference of antioxidants activity from different extraction techniques of Jerusalem artichoke (Helianthus tuberosus). Three different extraction techniques involved were aqueous extraction, autoclave-assisted extraction and $70 \%$ ethanol extraction. Total phenolic content (TPC) was determined using the FolinCiocalteu method. The antioxidant activities were analysed using ferric reducing antioxidant power (FRAP) and 2,2-diphenyl-1-picrylhydrazyl (DPPH). The autoclaveassisted extract showed the highest total phenolic content and both FRAP and DPPH activities at $127.87 \pm 0.08 \mathrm{mg} \mathrm{GAE} / \mathrm{g}, 53.38 \pm 2.76 \mathrm{mg}$ AAE/g and $79.92 \pm 0.26 \mathrm{mg}$ $\mathrm{AAE} / \mathrm{g}$, respectively. Meanwhile, the aqueous extracts showed 2-fold higher total phenolic content compared to the ethanol extracts at $65.73 \pm 9.44$ and $30.61 \pm 0.00 \mathrm{mg} \mathrm{GAE} / \mathrm{g}$. Conversely, antioxidant activities obtained from the ethanol extracts $(41.49 \pm 2.54$ and $41.10 \pm 0.10 \mathrm{mg} \mathrm{AAE} / \mathrm{g})$ were higher compared to the aqueous extracts $(23.29 \pm 0.47$ and $5.24 \pm 0.38 \mathrm{mg}$ AAE/g.). This study suggests that different extraction methods influenced the findings of total phenolic content and antioxidant activities of the Jerusalem artichoke. Among three different extraction methods, the results suggest that the effect of autoclaveassisted extraction is more effective for the antioxidant extraction from Jerusalem artichoke than aqueous and $70 \%$ ethanol extraction. Hence, Jerusalem artichoke can be considered as a potential source of natural antioxidants.
\end{abstract}

Keywords: aqueous extraction; autoclave-assisted extraction; ethanol extraction; FRAP; $\mathrm{DPPH}$

Received: $24^{\text {th }}$ November 2020

Received in revised form: $30^{\text {th }}$ December 2020

Accepted: $4^{\text {th }}$ January 2021

Citation: Nur Diyana, A., Puteh, F., Mohd Zulkifli, N. I., Hasnan, N. Z. N. Evaluation of total phenolic content and antioxidant activities from different extraction techniques of Helianthus tuberosus. Adv Agri Food Res J 2021; 2(1): a0000165. https://doi.org/10.36877/aafrj.a0000165

Available Online: $13^{\text {th }}$ January 2021 


\section{Introduction}

Free radicals have been identified as one of the primary factors in developing noncommunicable disorders such as cancer, ageing, diabetes and many other serious diseases. Antioxidants derived from vegetables may help the body in fighting against damages caused by these free radicals. Furthermore, it can protect oxidative stress from damaging organelle and cell membranes, as it has been reported in many studies (Catana et al., 2018). Common vegetables such as spinach, crucifers, broccoli flowers, kale, beans and alfalfa sprouts are known to have high antioxidants and have been incorporated in many diets and supplement (Aletor et al., 2002; Ali et al., 2010; Yadav, et al., 2013). Fruits such as orange, blueberries, strawberries, jack fruit and palm are also well-known as a good source of vitamin and high in phenolic contents that can act as antioxidants (Swami et al., 2012; Preira et al., 2013). Beside phenolic content, other antioxidant compounds that can be found in vegetables and fruits include flavonoids, isoflavonoids and anthocyanins (Nizioł-Łukaszewska et al., 2018).

Jerusalem artichoke, or scientifically known as Helianthus tuberosus have recently gained interest among researchers due to its potential health benefits. It is a perennial species belongs to Asterceae family, native to the Eastern North America region, closely related to the tall plant from the sunflower family. The tuber is larger and has few twigs and the taste is sweet and crispy. It is a rich source of carbohydrate and the content of inulin in its tuber can reach up to $85 \%$ when its dried, with moderate amount of protein and low in fat and sugar (Monti et al. 2005; Saengthongpinit \& Sajjaanantakul 2005; Tassoni et al. 2010). Jerusalem artichoke extract draws great attention to produce a variety of potential bioproducts. The extraction method is an initial step to separate the bioactive compound from plant and vegetable matrices. Current trends in Jerusalem artichoke extraction method are using organic solvent extraction to produce bioactive compounds and water extraction to produce functional food like inulin (Yang et al., 2015). This study describes three types of Jerusalem artichoke extraction methods, which chemical extractions using ethanol, aqueous and autoclave-assisted extraction. Aqueous extraction considered as the greenest solvent and have several advantages over solvent extraction such as safer operation, less cost and production of a variety of products due to its polarity resulted in high extract yield. Do et al. (2014) has described that ethanol known to be a good solvent for polyphenol extraction. Antioxidant compounds can be extracted more in less polar solvent like ethanol due to its polarity dependant characteristic. Meanwhile, autoclave-assisted extraction technique has been introduced and proven to give better results in bioactive extraction by utilizing the 
higher pressure and temperature compared to other methods (Park \& Hong, 2014; Suh et al., 2017)

Total phenolic content and antioxidants activities of all the extracts were measured using total phenolic content by Folin-Ciocalteu colorimetric method, 1-diphenyl-2-picrylhydrazil (DPPH) free radical scavenging activity and ferric reducing antioxidant power (FRAP). The FRAP (ferric reducing antioxidant power) assay is a measure of the compounds' presence with reducing power in the juice. The FRAP method relies on the reduction by the antioxidants, of the complex ferric ion-TPTZ (2,4,6-tri(2-pyridyl)-1,3,5-triazine). The binding of $\mathrm{Fe} 2+$ to the ligand produces a very strong navy-blue colour. The absorbance can be determined to test the amount of iron reduced and can be correlated with the content of antioxidants. Meanwhile, DPPH is a measure of the compound's presence with radical scavenging capacity. The DPPH assay was considered as a good method for determining antioxidant activity, since it is a stable free radical due to the delocalization of the spare electron on the whole molecule. The radical has a deep violet colour in solution with a strong absorption band centred at about $520 \mathrm{~nm}$. When DPPH reacts with a hydrogen donor (antioxidant), the reduced DPPH is generated without dimerize and therefore, visual monitoring of the reaction is enabled from the change in the optical absorption at $520 \mathrm{~nm}$. Hence, the method is proven to be sensitive and reliable (Zhao et al., 2015). The absorbance reduction depends linearly on the antioxidant concentration. Interestingly, several studies have highlighted the potential for Jerusalem artichoke to be the source of antioxidant in human's diet. (Nizioł-Łukaszewska et al., 2018)

Hence, it becomes our interest to investigate the most effective way to extract the bioactive compound from Jerusalem artichoke that can contribute to the increase in antioxidant intake for human consumption. Therefore, the extraction process should be critically selected to attain the desired bioactive substance and its activities of natural plant extracts. Jerusalem artichoke rich with antioxidants can be used as active ingredients for many purposes like combating regenerative and ageing properties. This paper aims to determine the difference of total phenolic content and antioxidant activities in different Jerusalem artichoke extraction methods. 


\section{Materials \& methods}

\subsection{Preparation of Raw Materials}

\subsubsection{Jerusalem artichoke preparation}

The Jerusalem artichoke tuber has been washed and sliced into a thin layer before being dried in a ventilated oven at $50{ }^{\circ} \mathrm{C}$ for 72 hours. The dried tubers were ground using ZM 200 Retsch variable speed rotor mill into a fine powder after the moisture content was measured at $5 \%$. The sample was kept cool and stored at $4{ }^{\circ} \mathrm{C}$ for further study. All samples in the form of tubers, dried sliced and powder were shown in Figure 1.

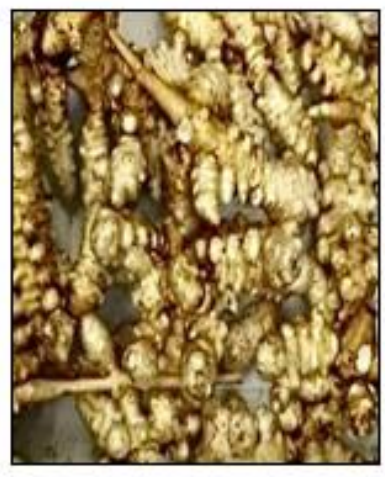

Jerusalem artichoke tubers

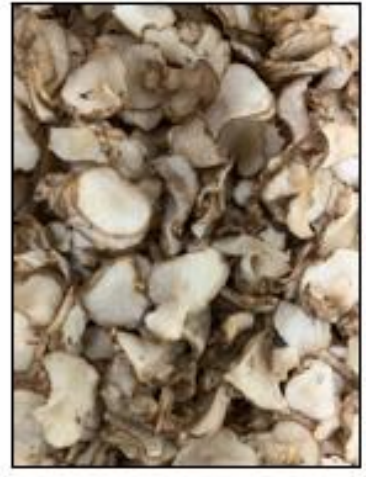

Dried sliced of Jerusalem artichoke

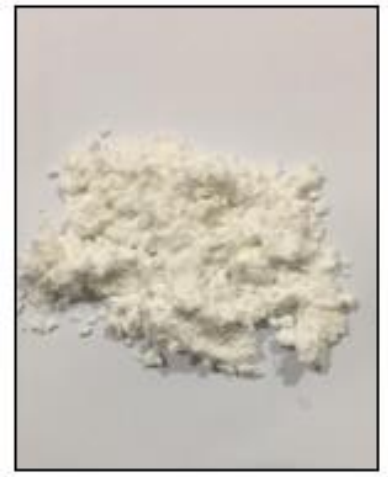

Jerusalem artichoke powder

Figure 1. Jerusalem artichoke preparation from tubers to powder.

2.2 The Chemical and Aqueous Extraction Method of Jerusalem Artichoke

\subsubsection{Ethanol extraction method}

Two grams of Jerusalem artichoke was added into $50 \mathrm{~mL} 70 \%$ ethanol (Vongsak, et al., 2013 and Alara et al., 2020) and further incubated at room temperature with $160 \mathrm{rpm}$ for $60 \mathrm{~min}$ in an incubator shaker (Innova 4000, New Brunswick Scientific, Canada). The mixture was then centrifuged at $20^{\circ} \mathrm{C}, 19712 \mathrm{G}$-force for $10 \mathrm{~min}$ before being filtered using Whatman no. 1 filter paper to separate the residue. All experiments were done in triplicates.

\subsubsection{Aqueous extraction method}

Two grams of Jerusalem artichoke powder was added into $50 \mathrm{~mL}$ distilled water and further incubated at room temperature with $160 \mathrm{rpm}$ for $60 \mathrm{~min}$ in an incubator shaker (Innova 4000, New Brunswick Scientific, Canada). Afterwards, the mixture was then 
centrifuged at $20^{\circ} \mathrm{C}, 19712 \mathrm{G}$-force for 10 minutes before filtered using Whatman no. 1 filter paper. All experiments were done in triplicates.

\subsection{Physical Extraction Method of Jerusalem Artichoke}

\subsubsection{Autoclave-assisted extraction method}

Approximately, 2 grams of Jerusalem artichoke powder was extracted using $50 \mathrm{~mL}$ distilled water. The mixture was heated to extract out the chemical compounds from the sample at $121^{\circ} \mathrm{C}$ with $0.10 \mathrm{MPa}$ using autoclave ES-315, TOMY, Japan. This method was slightly modified according to Suh et al. (2017). The extract was centrifuged at $20^{\circ} \mathrm{C}, 19712$ G-force for $10 \mathrm{~min}$ before filtered using Whatman no. 1 filter paper. All experiments were done in triplicates.

\subsection{Total Phenolic Content (TPC) By Folin-Ciocalteu Colourimetric Method}

About $1 \mathrm{~mL}$ Jerusalem artichoke extracts were mixed with $5 \mathrm{~mL}$ Folin-Ciocalteu reagent and incubated at ambient room temperature for five min. A total of $4 \mathrm{~mL}$ of $7.5 \%$ of the sodium carbonate solution were added and vortexed. The mixture was incubated in the dark place for 120 minbefore measured the absorbance at $765 \mathrm{~nm}$ against the reagent blank using Varian Cary 50 Conc. UV-Vis spectrophotometer, Varian Inc., USA (Koh et al., 2012). The sample was incubated in the dark place because the reaction was sensitive to light in the presence of the Folin-Ciocalteu reagent. The amount of total phenolic content was expressed as Gallic acid equivalents (mg GAE/g extract) from a calibration curve.

\subsection{Antioxidant Activity}

\subsubsection{Determination of free radical scavenging activity (DPPH)}

The DPPH activity was measured as described by Koh et al. (2012). The aliquots of $150 \mu \mathrm{L}$ were mixed with freshly prepared of $2850 \mu \mathrm{L}$ of 1,1-diphenyl-2-picryl-hydrazil (DPPH) methanolic solution and vortexed. The mixture was then incubated in dark condition for 30 minutes at room temperature. The absorbance was read at $515 \mathrm{~nm}$ using Varian Cary 50 Conc. UV-Vis spectrophotometer, Varian Inc., USA and ascorbic acid was used as a standard to set up DPPH calibration curve. 


\subsubsection{Determination of ferric reducing antioxidant power (FRAP)}

Aliquots of $150 \mu \mathrm{L}$ of the extract were mixed with $2850 \mu \mathrm{L}$ of freshly prepared FRAP reagent. The mixture was incubated in dark condition for $30 \mathrm{~min}$ at room temperature. The FRAP values were measured by comparing the absorbance change of blue coloured ferroustripyridyltriazine complex at $593 \mathrm{~nm}$ using Varian Cary 50 Conc. UV-Vis spectrophotometer, Varian Inc., USA. The results were determined from ascorbic acid calibration curve.

\subsection{Statistical and Data Analysis}

The data collected were analysed using Minitab Software Version 18, Minitab, LLC. The differences between different extracts were represented as mean value \pm standard deviation $(n=3)$ and compared using one- way analysis of variance (ANOVA) followed by Turkey's test at a significance level of $p<0.05$.

\section{Results}

The result showed that the autoclave-assisted extracts exhibited the highest total phenolic content and antioxidants activities with $127.87 \pm 0.08 \mathrm{mg} \mathrm{GAE} / \mathrm{g}, 79.92 \pm 0.26 \mathrm{mg}$ $\mathrm{AAE} / \mathrm{g}$ and $53.38 \pm 2.76 \mathrm{mg} \mathrm{AAE} / \mathrm{g}$, respectively (Figure 2). It was further observed that the total phenolic content of Jerusalem artichoke is two-fold higher in the autoclave-assisted extracts compared to the aqueous and four-fold than ethanol extracts at $127.87 \pm 0.08,65.73$ \pm 9.44 and $30.61 \pm 0.00 \mathrm{mg} \mathrm{GAE} / \mathrm{g}$ with significant difference at $p<0.05$. Jerusalem artichoke using autoclave-assisted extraction method indicates much higher activity to scavenge free radicals of 2,2-Diphenyl-1-picryl-hydrazylhydrate (DPPH) compared to the ethanol and aqueous extraction at 79.92 $\pm 0.26,41.10 \pm 0.10$ and $5.24 \pm 0.38 \mathrm{mg}$ AAE/g, respectively (Figure 3). A similar tendency was also observed in ferric reducing antioxidant power (FRAP) of different Jerusalem artichoke extracts in Figure 4. The highest ferric reducing antioxidant power (FRAP) activity was obtained in the autoclave-assisted extract at $53.38 \pm$ $2.76 \mathrm{mg}$ AAE/g followed by ethanol extract at $41.49 \pm 2.54 \mathrm{mg} \mathrm{AAE} / \mathrm{g}$ and aqueous extract at $23.29 \pm 0.47 \mathrm{mg} \mathrm{AAE} / \mathrm{g}$ with significant different at $p<0.05$. 


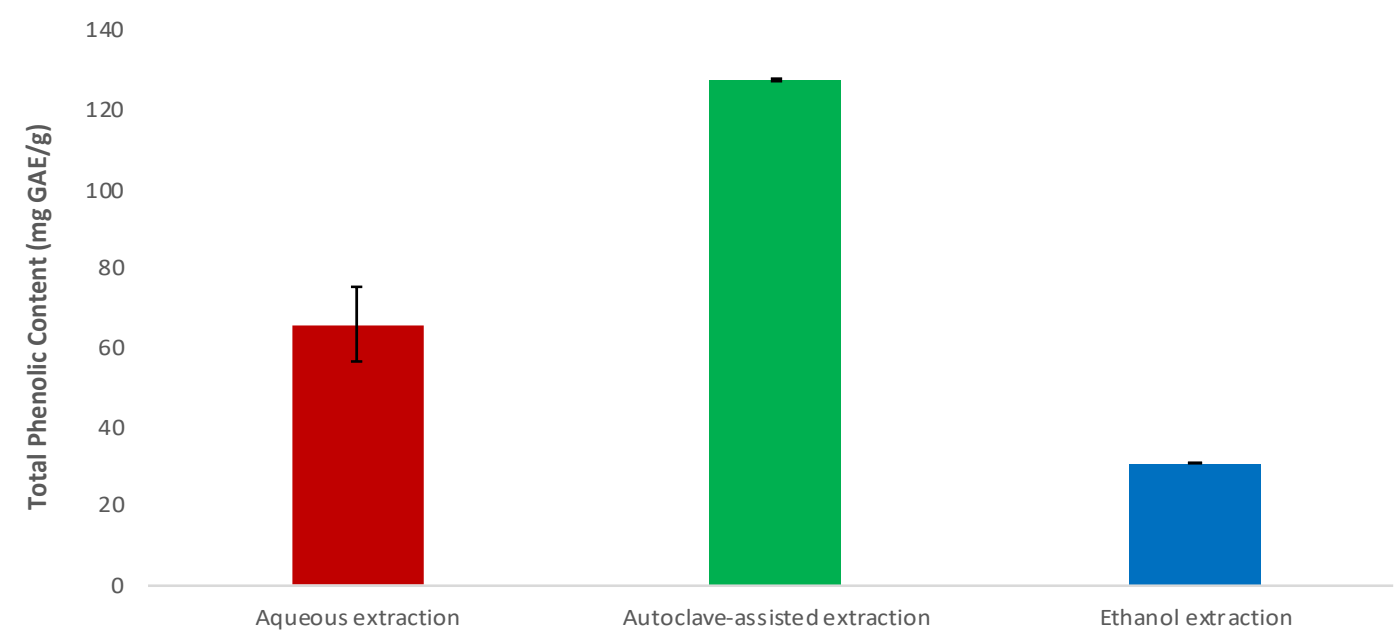

Figure 2. Total phenolic content (TPC) of different Jerusalem artichoke extracts.

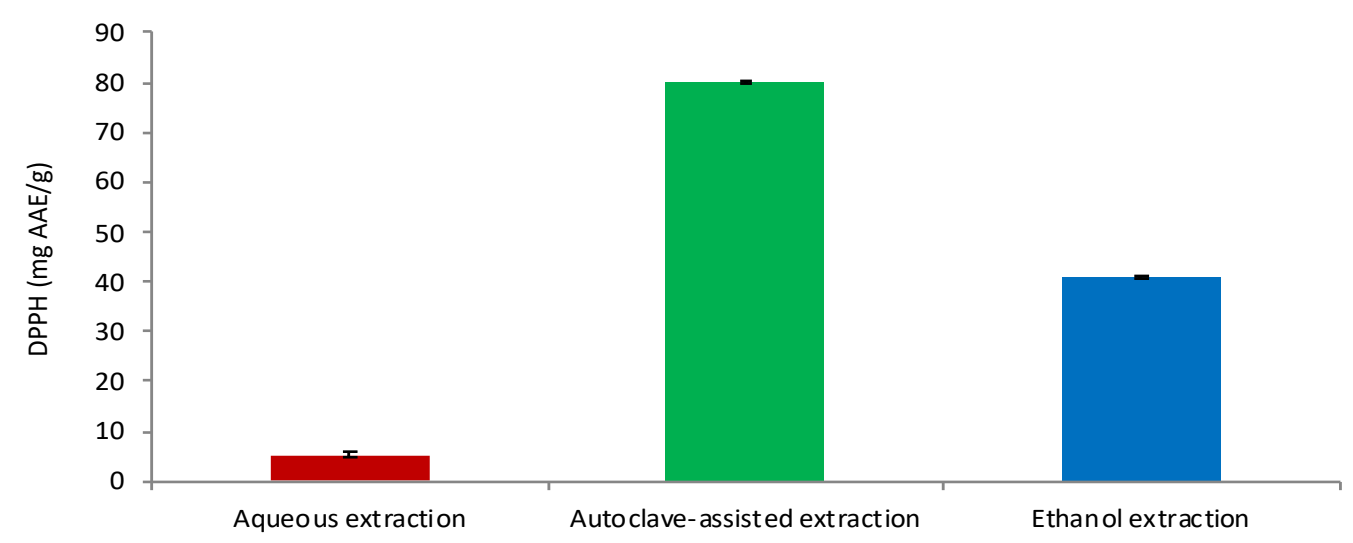

Figure 3. 2,2-Diphenyl-1-picryl-hydrazylhydrate (DPPH) of different Jerusalem artichoke extracts.

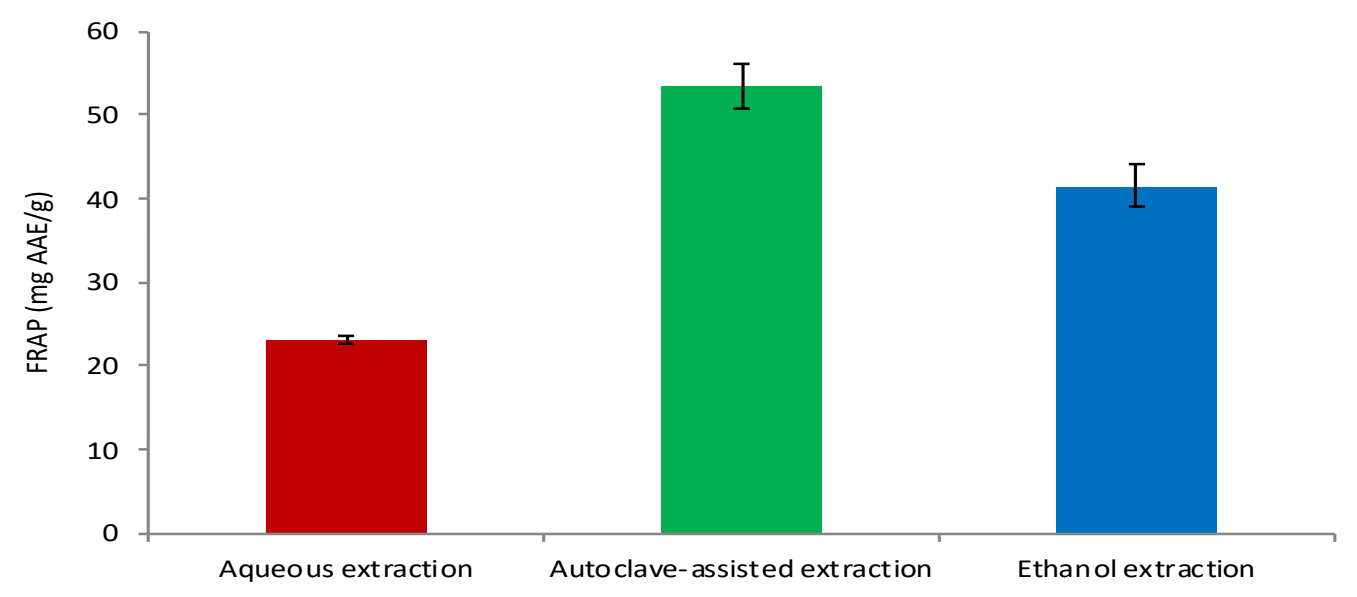

Figure 4. Ferric reducing antioxidant power (FRAP) of different Jerusalem artichoke extracts. 


\section{Discussion}

In this study, the total phenolic content and antioxidant activities of Jerusalem artichoke extracts as obtained from the aqueous, autoclave-assisted and ethanol extraction, were measured based on DPPH and FRAP assays. The different extraction method of Jerusalem artichoke contributes to different total phenolic content and antioxidants activities. Phenolic compounds can be found in complex carbohydrate and protein. The composition of phenolic fraction in natural plant sources can be dependent on the species, variety, agrotechnical and climate conditions and particularly on their plant part (Nizioł-Łukaszewska et al., 2018). According to Yuan et al. (2012), the Jerusalem artichoke tubes contains high chlorogenic (Showkat et al., 2019) and dicaffeic acid. The polyphenolic compound in Jerusalem artichoke extracts plays a significant role in their antioxidant capacity. The high amount of total phenolic content has reduced free radicals. Catana et al. (2018) reported on the high values of phenolic compound in Jerusalem artichoke powders in the range of 20.30 to $44.03 \mathrm{mg} \mathrm{GAE} / \mathrm{g}$ that correspond to the antioxidant values of 1.78 to $6.04 \mathrm{mg}$ Trolox Equivalent/g. Comparatively, this value is significantly higher than the reported amounts of polyphenol content by Petkova et al. (2014) in Jerusalem artichoke flour as 12 to $16 \mathrm{mg}$ $\mathrm{GAE} / \mathrm{g}$ and Inchuen et al. (2014) as 2.5 to 5.3 for the blanched Jerusalem artichoke tuber. However, Zhang and Kim, (2015) reported the similar findings on low total phenolic content of ethanolic Jerusalem artichoke extract at $15.48 \pm 0.33$ AAE ug/mL compared to water extracts at $18.48 \pm 0.22 \mathrm{AAE} \mathrm{ug} / \mathrm{mL}$.

Jerusalem artichoke using autoclave-assisted extract indicates much higher activity to scavenge free radicals of 2,2-diphenyl-1-picryl-hydrazyl (DPPH) compared to the ethanol and aqueous extraction at 79.92 $\pm 0.26,41.10 \pm 0.10$ and $5.24 \pm 0.38 \mathrm{mg}$ AAE/g, respectively (Figure 3). A similar tendency was also observed in ferric reducing antioxidant power (FRAP) of different Jerusalem artichoke extracts in Figure 4. The highest ferric reducing antioxidant power (FRAP) activity was obtained in the autoclave-assisted extract at $53.38 \pm$ $2.76 \mathrm{mg}$ AAE/g followed by ethanol extract at $41.49 \pm 2.54 \mathrm{mg} \mathrm{AAE} / \mathrm{g}$ and aqueous extract at $23.29 \pm 0.47 \mathrm{mg} \mathrm{AAE} / \mathrm{g}$ with significant different at $p<0.05$. Besides, several studies have found a correlation between reducing power and capacity of antioxidant in plant matrices extracts (Lee et al., 2003). These correlated results in Figure 2, 3 and 4 for the autoclaveassisted extraction indicates that the total phenolic content is the determinant of the antioxidant activity in the Jerusalem artichoke extract. High antioxidant activity for the thermally processed Jerusalem artichoke could be explained by the increased amount of phenols from their matrix. The high temperature disrupts the cell membranes and cell walls 
and releases phytochemicals from the Jerusalem artichoke, which increases the pool of phenols. Autoclave-assisted extraction is operationally an advantage allowing shorter extraction time and permeable at a higher temperature, where it can enhance the contact between substrate and extractant, thus increasing the antioxidant yield. According to Jing \& Kitts, 2000, this application has proven to enhance the antioxidant activity of lysine residues in peptide. These results are similar with several studies that demonstrated the autoclave extraction to be more efficient compared to the water extraction due to high temperature that contributes towards escalating capacity of water to extract the compound by enhancing their solubility through decreasing the surface tension (Suh et al., 2017). The finding is interesting, as commonly the thermally processed agriculture products have long been associated with lower nutritional value compared with non-thermal treatment. This consideration is however, based on the fact that vitamin $\mathrm{C}$ or other thermolabile compounds may lose their activity due to oxidation or in consequences of leaching into the water during cooking or blanching. Nevertheless, it is important to note that a rise in extraction temperature may break the phenolic-matrix bonds and influence the membrane structure of plant cells. Thus, more antioxidants can be obtained and contribute to higher added-value extracts. Although different type of extraction techniques produced a different amount of antioxidant, they also produced different bioactive compounds that contributed to the antioxidant activities (Selvamuthukumaran \& Shi, 2017). To obtain a greater understanding of the antioxidant properties of the Jerusalem artichoke, their secondary metabolites and its phenolic compounds in all extracts can be further profiled.

\section{Conclusion}

This study demonstrates that Jerusalem artichoke extracts are rich in total phenolic content and antioxidant activities. Autoclave-assisted extract of Jerusalem artichoke presented the highest total phenolic content and antioxidant activities compared to ethanol and aqueous extracts. The high activity of antioxidant extracts indicates that Jerusalem artichoke may have potential as a bio-ingredient and a natural antioxidant. The findings also contradict the notion that processed agriculture produces have lower nutritional values than their processed counterparts at lower temperature and suggests that the effect of autoclaveassisted extraction is more effective for the Jerusalem artichoke than aqueous extraction and $70 \%$ ethanol extraction in releasing phenols from their matrix, whereby the phenols are responsible for the high antioxidant activity. Secondary metabolites profiling of the extracts should be further investigated to comprehend the antioxidant properties in Jerusalem artichoke in the future. This work could have a direct influence on consumers' food selection 
by increasing their awareness on the health benefits of processed agriculture produces and encouraging them to obtain their phytochemical sources from a variety of sources, processed by different techniques.

Funding: This study was supported by Putra Grant 9677900.

Acknowledgements: The authors would like to thank many people who have contributed either directly or indirectly in this study. Special thanks to Mohd Zulkifli Mohd Zainudin, Shahril and Dr. Md Selamat for their continuous support, professional guidance and encouragement throughout the study.

Conflicts of Interest: The authors declare no conflict of interest.

\section{References}

Aletor, O., Oshodi, A. A., Ipinmoroti, K. (2002). Chemical composition of common leafy vegetables and functional properties of their leaf protein concentrates. Journal of Food Chemistry, 78, 63-68. doi: https://doi.org/10.1016/S0308-8146(01)00376-4.

Ali, M. B., Khandaker, L., Oba. S. (2010). Changes in pigments, total polyphenol, antioxidant activity and color parameters of red and green edible amaranth leaves under different shade levels. Journal of Food, Agriculture \& Environment, 8(3-4), 217-222.

Catană, L., Catană, M., Iorga, E., et al. (2018). Valorification of Jerusalem Artichoke tubers (Helianthus Tuberosus) for achieving of functional ingredient with high nutritional value. In: Agriculture for life. Life for Agriculture Conference Proceedings, 1(1), 276-283. doi: https://doi.org/10.2478/alife-20180041.

Do, Q. D., Angkaijaya, A. E., Tran-Nguyen, P. L., et al. (2014). Effect of extraction solvent on total phenol content, total flavonoid content and antioxidant activity of Limnophila aromatica. Journal of Food and Drug Analysis, 22, 296-302. doi: http://dx.doi.org/10.1016/j.jfda.2013.11.001.

Inchuen, S., Porniammongkol, O., Duangkhamchan, W. (2014). Effect of drying methods on chemical and functional properties of Jerusalem Artichoke (Helianthus tuberosus L.) powders. Advanced Materials Research, 931-932, 1512-1517.

Jing, H., Kitts, D. D. (2000). Comparison of the antioxidative and cytotoxic proper- ties of glucose-lysine and fructose-lysine Maillard reaction products. Food Research International, 33, 509-516. doi: https://doi.org/10.1016/S0963-9969(00)00076-4

Koh, S. P., Jamaluddin, A., Alitheen, N. B, et al. (2012). Nutritional values of tempe inoculated with different strains of Rhizophus: its y-aminobutyric acid content and antioxidant property. Journal of Tropical Agriculture and Food Science, 40(2), 181-192.

Lee, K.W., Kim, Y.J. Kim, D. O., et al. (2003). Major phenolics in apple and their contribution to the total antioxidant capacity. Journal of Agricultural and Food Chemistry, 51, 6516-6520. doi: https://doi.org/10.1021/jf034475w.

Monti, A., Amaducci, M. T., Venturi, G. (2005). Growth response, leaf gas exchange and fructans accumulation of Jerusalem artichoke (Helianthus tuberosus L.) as affected by different water regimes. European Journal of Agronomy, 23(2), 136-145. doi: https://doi.org/10.1016/j.eja.2004.11.001 
Nizioł-Łukaszewska, Z., Furman-Toczek, D., Zagorska-Dziok. (2018). Antioxidant activity and cytotoxicity of Jerusalem artichoke tubers and leaves extracts on $\mathrm{HaCaT}$ and $\mathrm{BJ}$ fibroblast cells. Lipids in Health and Disease, 17, 280.

Park, H. M., Hong, J. H. (2014). Antioxidant activity of extracts with extraction methods from Phellinus linteus mycelium on Mori ramulus. Korean Journal on Food Preservation, 21, 565-572. doi: 10.11002/kjfp.2014.21.4.565.

Pereira, M. C., Steffens, R. S., Jablonski, A., et al. (2013). Characterization, bioactive compounds and antioxidant potential of three Brazilian fruits. Journal of Food Composition and Analysis, 29, 19-24. doi: https://doi.org/10.1016/j.jfca.2012.07.013

Petkova, N., Ivanov, I., Denev, P., et al. (2014). Bioactive substance and free radical scavenging activities of flour from Jerusalem Artichoke (Helianthus tuberosus L.) Tubers: a comparative study. Turkish Journal of Agricultural and Natural Sciences, Special Issue-2, 1773-1778.

Saengthongpinit, W., Sajjaanantakul, T. (2005). Influence of harvest time and forage temperature on characteristic of inulin from Jerusalem artichoke (Helianthus tuberosus L.) tubers. Postharvest Biology and Technology, 37, 93-100. doi: https://doi.org/10.1016/j.postharvbio.2005.03.004

Selvamuthukumaran, M., Shi, J. (2017). Recent advances in extraction of antioxidants from plant by-products processing industries. Food Quality and Safety, 1, 61-81. doi: https://doi.org/10.1093/fqsafe/fyx004

Showkat, M. M., Falck-Ytter, A. B., Straetkvern, K. O. (2019). Phenolic acids in Jerusalem artichoke (Helianthus tuberosus L.): Plant organ dependent antioxidant activity and optimized extraction from leaves. Molecules, 24, 3296. doi: https://doi.org/10.3390/molecules24183296

Suh, S., Kim, Y. E., Yang, H., et al. (2017). Influence of autoclave treatment and enzymatic hydrolysis on the antioxidant activity of Opuntia ficus-indica fruit extract. Food Science and Biotechnology, 26(3), 581590. doi: https://doi.org/10.1007/s10068-017-0085-3

Swami, S. B., Thakor, N. J., Haldankar, P. M. et al. (2012). Jackfruit and its many functional components as related to human health: A Review: Comprehensive Reviews. Food Science and Food Safety, 11, 565576. Doi: https://doi.org/10.1111/j.1541-4337.2012.00210.x

Tassoni, A., Bagni, N., Ferri, M., et al. (2010). Helianthus tuberosus and polyamine research: Past and recent applications of a classical growth model. Plant Physiology and Biochemistry, 48(7), 496-505. doi: http://doi.org/10.1016/j.plaphy.2010.01.019

Vongsak, B., Sithisarn, P., Mangmool, S. et al., (2013). Maximizing total phenolics, total flavonoids contents and antioxidant activity of Moringa oleifera leaf extract by the appropriate extraction method. Industrial Crops and Products, 44, 566-571. doi: http://doi.org/10.1016/j.indcrop.2012.09.021

Yadav, R. K., Subodh, J., Raj, K., et al. (2013). Studies on diverse genotypes of amaranth (Amaranthus spp.) for their antioxidant and nutritional activities. Indian Journal of Agricultural Sciences, 83(1), 83-86.

Yang, L., He, Q. S., Corscadden, K. et al. (2015). The prospects of Jerusalem artichoke in functional food ingredients and bioenergy production. Biotechnology reports, 5, 77-88. doi: http://doi.org/10.1016/j.btre.2014.12.004 
Yuan, X., Gao, M., Xiao, H., et al. (2012). Free radical scavenging activities and bioactive substances of Jerusalem artichoke (Helianthus tuberosus L.) leaves. Food Chemistry, 133, 10-14. doi: http://doi.org/10.1016/j.foodchem.2011.09.071

Zhang, Q., Kim, H. Y. (2015). Antioxidant, anti-inflammatory and cytotoxicity on human lung epithelial A549 cells of Jerusalem artichoke (Helianthus tuberosus L) tuber. Korean Journal of Plant Resources, 28(3), 305-311. doi: http://dx.doi.org/10.7732/kjpr.2015.28.3.305

Zhao, Y., Wu, Y. Z., Wang, M. (2015). Bioactive substances of plant origin. In: Cheung P, Mehta B, (Eds.) Handbook of food chemistry. Heidelberg, Berlin. Springer. doi: https://doi.org/10.1007/978-3-64241609-5_13-1

Copyright $\odot 2020$ by Nur Diyana A, et al. and HH Publisher. This work is licensed under the Creative Commons Attribution-NonCommercial 4.0 International Lisence (CC-BY-NC4.0) 